\title{
PRELIMINARY STUDY OF THE STELLAR POPULATIONS AND DENSITY PROFILE OF NGC 6624 USING HST
}

\author{
PURAGRA GUHATHAKURTA \\ Univ. of California, Lick Obs., Santa Cruz, CA 95064, USA \\ BRIAN YANNY \\ Fermi National Accelerator Lab., Batavia, IL 60510, USA \\ JOHN N. BAHCALL \\ Inst. for Advanced Study, Princeton, NJ 08540, USA \\ AND \\ DONALD P. SCHNEIDER \\ Dept. of Astronomy \& Astrophysics, Pennsylvania State Univ., \\ University Park, PA 16802, USA
}

We present preliminary results from an ongoing study of the central $5 \operatorname{arcmin}^{2}$ of NGC 6624 based on short F336W, F439W, and F555W (UBV) Hubble Space Telescope WFPC2 exposures (Yanny et al. 1996). NGC 6624 is a dense, metal rich, post core collapse globular cluster at a distance of $8.1 \mathrm{kpc}$. Nearly 5000 stars with $V \lesssim 21$ (1.5 mag below the turnoff) are detected within the $34^{\prime \prime} \times 34^{\prime \prime}$ area of the PC1 CCD which imaged the cluster center. Individual stars brighter than $V=20$ are easily identifiable in the central image section shown in Fig. 1. Image simulations and similar data on the denser cluster M15 indicate that the effects of incompleteness and photometric error are negligible on the $V<19.5$ sample of stars in NGC 6624. The stellar surface density profile derived from such a sample is well approximated by a power law of index $\alpha=-0.85$ (Fig. 2). The density profile shows no hint of flattening towards smaller radii in the radial range over which it is reliably measured $(r \gtrsim 0$ !"3). A $B$ vs. $U-V$ color-magnitude diagram of the $r<15^{\prime \prime}$ region (Fig. 3 ) shows a well defined blue straggler sequence. Our preliminary findings are consistent with an earlier study of this cluster by Sosin and King (1995) using pre-repair FOC images.

\section{References}

Sosin, C. and King, I.R. (1995) A. J. 109, 639. 


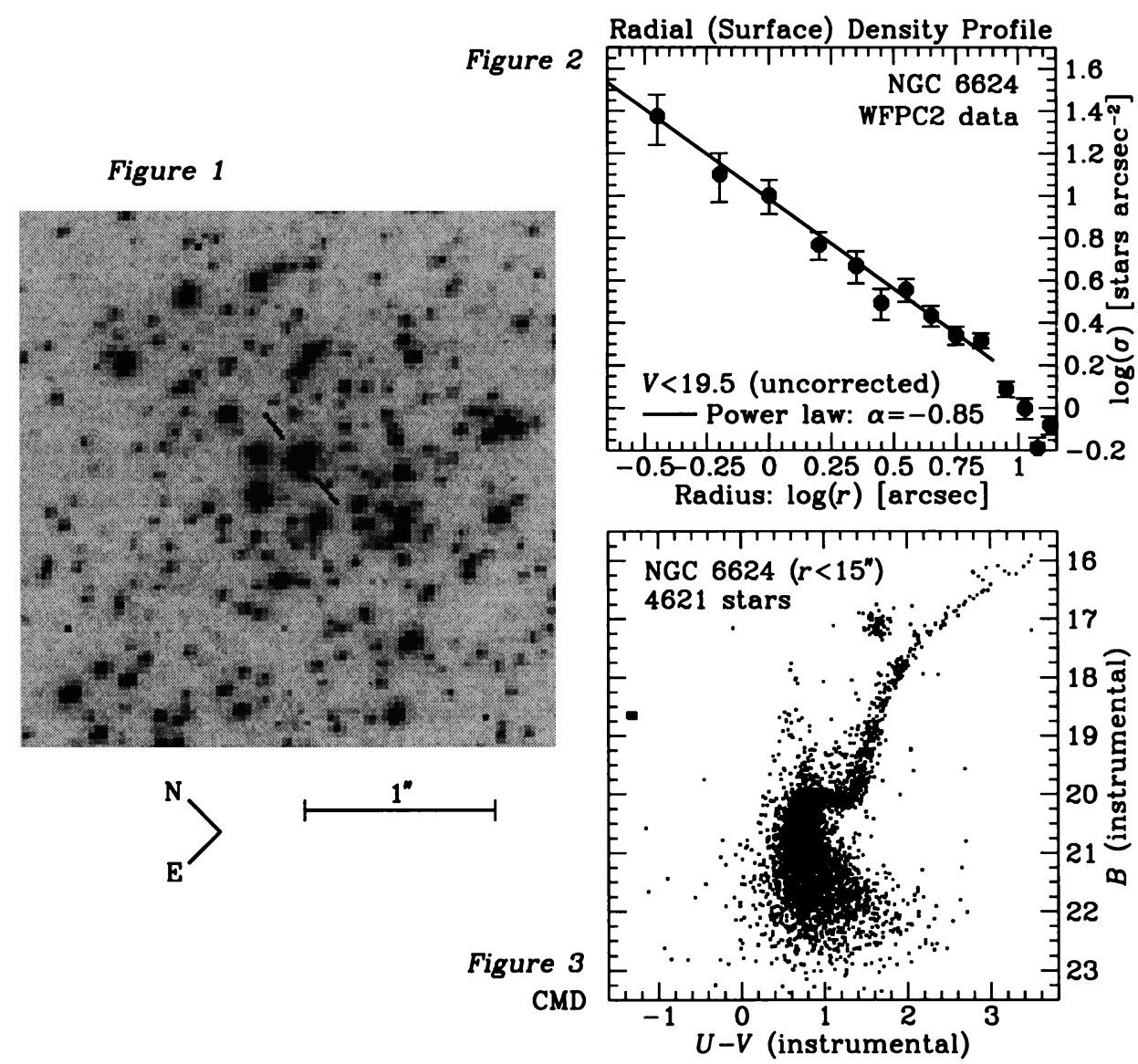

Figure 1. A $2 . .5 \times 2.5$ section of a $U$ band HST WFPC2 exposure of NGC 6624 , centered on the X-ray source $4 \mathrm{U}$ 1820-30 (marked by line segments). The cluster centroid lies about 0.5 south of this object. Individual stars are easily resolved in this 0.1 -resolution image. The number density of stars is high enough to permit reliable determination of the density distribution on subarcsecond scales.

Figure 2. The projected density profile of $V<19.5$ stars in NGC 6624. The solid line shows an $\alpha=-0.85$ power law and it is a good fit to the data. The data do not require a finite core; the $99 \%$ upper limit on the core radius is about $2^{\prime \prime}(0.08 \mathrm{pc})$.

Figure 3. A $(B, U-V)$ color-magnitude diagram of over 4500 stars within $r<15^{\prime \prime}$ of NGC 6624's center. The sample is complete down to $B \sim 21.5$ which is about 1.5 mag below the main sequence turnoff. Note the clear sequence of blue stragglers in the region $18<B<19.75$ and $0.4<U-V<0.9$. The bold box near the left edge of the plot marks the X-ray bright object $4 \mathrm{U} 1820-30$.

Yanny, B., Guhathakurta, P., Schneider, D.P. and Bahcall, J.N. (1996) A. J. in prep. 